\title{
Article \\ On Fourier Coefficients of the Symmetric Square L-Function at Piatetski-Shapiro Prime Twins
}

\author{
Xue Han, Xiaofei Yan and Deyu Zhang *
}

check for

updates

Citation: Han, X.; Yan, X.; Zhang, D.

On Fourier Coefficients of the

Symmetric Square L-Function at

Piatetski-Shapiro Prime Twins.

Mathematics 2021, 9, 1254. https:/ /

doi.org/10.3390/math9111254

Academic Editor: Luis Dieulefait

Received: 28 April 2021

Accepted: 26 May 2021

Published: 30 May 2021

Publisher's Note: MDPI stays neutral with regard to jurisdictional claims in published maps and institutional affiliations.

Copyright: (c) 2021 by the authors. Licensee MDPI, Basel, Switzerland. This article is an open access article distributed under the terms and conditions of the Creative Commons Attribution (CC BY) license (https:// creativecommons.org/licenses/by/ $4.0 /)$.
School of Mathematics and Statistics, Shandong Normal University, Jinan 250358, China; han_xue@stu.sdnu.edu.cn (X.H.); xfyan@sdnu.edu.cn (X.Y.)

* Correspondence: zhangdeyu@sdnu.edu.cn

Abstract: Let $\mathbb{P}_{c}(x)=\left\{p \leq x \mid p,\left[p^{c}\right]\right.$ are primes $\}, c \in \mathbb{R}^{+} \backslash \mathbb{N}$ and $\lambda_{\text {sym } m^{2} f}(n)$ be the $n$-th Fourier coefficient associated with the symmetric square $L$-function $L\left(s, s y m^{2} f\right)$. For any $A>0$, we prove that the mean value of $\lambda_{\text {sym }}{ }^{2} f(n)$ over $\mathbb{P}_{c}(x)$ is $\ll x \log ^{-A-2} x$ for almost all $c \in(\varepsilon,(\sqrt{5}+3) / 8-\varepsilon)$ in the sense of Lebesgue measure. Furthermore, it holds for all $c \in(0,1)$ under the Riemann Hypothesis. Furthermore, we obtain that asymptotic formula for $\lambda_{f}^{2}(n)$ over $\mathbb{P}_{c}(x)$ is $\sum_{p, q \text { prime } p \leq x, q=\left[p^{c}\right]} \lambda_{f}^{2}(p)=$ $\frac{x}{c \log ^{2} x}(1+o(1))$, for almost all $c \in(\varepsilon,(\sqrt{5}+3) / 8-\varepsilon)$, where $\lambda_{f}(n)$ is the normalized $n$-th Fourier coefficient associated with a holomorphic cusp form $f$ for the full modular group.

Keywords: primes; Fourier coefficient; symmetric square $L$-function

MSC: 11A41; 11F11; 11F30

\section{Introduction}

Let $k$ be an even positive integer, $f$ be a holomorphic cusp form of weight $k$ for the full modular group and $\lambda_{f}(n)$ be the normalized $n$-th Fourier coefficient of $f$,i.e.,

$$
f(z)=\sum_{n=1}^{\infty} \lambda_{f}(n) n^{\frac{k-1}{2}} e(n z) .
$$

If we assume that $f$ is an eigenform of all the Hecke operators, then $f$ can be normalized such that $\lambda_{f}(1)=1$ and $\lambda_{f}(n)$ is real. We define the Hecke $L$-function associated to $f$ for $\Re s>1$ by

$$
L(s, f)=\sum_{n=1}^{\infty} \frac{\lambda_{f}(n)}{n^{s}}=\prod_{p}\left(1-\lambda_{f}(p) p^{-s}+p^{-2 s}\right)^{-1} .
$$

For any prime $p$ and all integers $v \geq 0$, we have

$$
\lambda_{f}\left(p^{v}\right)=\alpha_{f}(p)^{v}+\alpha_{f}(p)^{v-1} \beta_{f}(p)+\cdots+\beta_{f}(p)^{v},
$$

where $\alpha_{f}(p), \beta_{f}(p)$ are the local parameters of $L(s, f)$ at prime $p$, satisfying

$$
\alpha_{f}(p)+\beta_{f}(p)=\lambda_{f}(p), \alpha_{f}(p) \beta_{f}(p)=1 \text {. }
$$

Then we have

$$
\lambda_{f}^{2}(p)=1+\lambda_{f}\left(p^{2}\right) .
$$

Deligne [1,2] proved Ramanujan-Petersson conjecture, i.e.,

$$
\left|\lambda_{f}(n)\right| \leq d(n) \ll n^{\varepsilon}
$$

for all $n \geq 1$, where $d(n)=\sum_{d \mid n} 1$. 
In order to detect the sign changes of $\lambda_{f}(n)$, many authors have studied the mean value of $\lambda_{f}(n)$ and obtained some good results. For example, see [1,3-22]. In addition, the sums of $\lambda_{f}(n)$ over primes have also been studied. It is known that (see for example Section 5.6 of Iwaniec and Kowalski [23]) there exists a constant $C>0$ such that

$$
\sum_{p \leq N} \lambda_{f}(p) \ll_{f} N \exp (-C \sqrt{\log N})
$$

The upper bound of (1) may reach $N^{\frac{1}{2}+\varepsilon}$, assuming the Riemann Hypothesis. Furthermore, we can establish that

$$
\sum_{p \leq N} \lambda_{f}^{2}(p) \sim \frac{N}{\log N}, \text { as } N \rightarrow \infty,
$$

by using the analytic properties of the Rankin-Selberg $L$-function $L(s, f \otimes \bar{f})$.

Another interesting question considered by many authors is the mean value of $\lambda_{f}(n)$ over certain sets of primes. For example, Baier and Zhao [24] studied the distribution of $\lambda_{f}(n)$ at Piatetski-Shapiro primes by considering estimates of exponential sum involving Hecke eigenvalue. Moreover, they conjectured that, for $1<c<1+\theta$ with some suitable $\theta>0$, there exists a constant $c_{f}>0$ such that

$$
\sum_{\substack{n \leq N \\\left[n^{c}\right] \in \mathbb{R}}} \lambda_{f}^{2}\left(\left[n^{c}\right]\right) \sim c_{f} \frac{N}{c \log N}, \text { as } N \rightarrow \infty
$$

Furthermore, we can define Piatetski-Shapiro prime twins if $p, q$ are primes and $q=\left[p^{c}\right]$, for any fixed $c \in \mathbb{R}^{+} \backslash \mathbb{N}$. Balog [25] and Dufner [26] proved that

$$
\sum_{\substack{p, q \text { prime } \\ p \leq x, q=\left[p^{c}\right]}} 1=\frac{x}{c \log ^{2} x}(1+o(1)), x \rightarrow \infty,
$$

for almost all $c \in(0,1)$ in the sense of Lebesgue measure. Furthermore, assuming the Riemann Hypothesis of automorphic $L$-function $L(s, f)$ is true, they found that (2) holds for all $c \in(0,1)$. Furthermore, Zhang and Zhai [27] studied the mean value of $\lambda_{f}(n)$ over Piatetski-Shapiro prime twins.

Motivated by the above results, we are interested in the distribution of $\lambda_{f}^{2}(n)$ at Piatetski-Shapiro prime twins. For the form $f$, we know the $L\left(s, s y m^{2} f\right)$ is an $L$-function for some $G L(3, \mathbb{Z})$ automorphic representation, which is often called the symmetric-square lift of $f$. The $n$-th Fourier coefficient of $L\left(s, s y m^{2} f\right)$ satisfies

$$
\lambda_{s y m^{2} f}(n)=\sum_{m l^{2}=n} \lambda_{f}\left(m^{2}\right) .
$$

The symmetric square $L$-function associated to $f$ is defined by

$$
L\left(s, s^{2} f\right)=\prod_{p} \prod_{0 \leq j \leq 2}\left(1-\frac{\alpha_{f}(p)^{2-j} \beta_{f}(p)^{j}}{p^{s}}\right)^{-1}=\sum_{n=1}^{\infty} \frac{\lambda_{s y m^{2} f}(n)}{n^{s}}
$$

in the half-plane $\Re_{s}>1$. Then, for all $n \geq 1, \lambda_{s y m^{2} f}(n)$ is also multiplicative, real and

$$
\left|\lambda_{\text {sym }^{2} f}(n)\right| \leq d_{3}(n)
$$

where $d_{3}(n)=\sum_{m \mid n} d(m)$. Furthermore, for all primes $p$, we have

$$
\lambda_{s y m^{2} f}(p)=\lambda_{f}\left(p^{2}\right) .
$$


Many authors studied the mean value of $\lambda_{\text {sym } m^{2} f}(n)$. For example, see References [28-36]. In this paper, we consider the mean value of Fourier coefficients of symmetric square $L$ function over Piatetski-Shapiro prime twins and obtain the following results, which imply a result on the distribution of $\lambda_{f}^{2}(n)$ at Piatetski-Shapiro prime twins.

Theorem 1. For almost all $c \in(\varepsilon,(\sqrt{5}+3) / 8-\varepsilon)$ and any $A>0$, we have

$$
\sum_{\substack{p, q \text { prime } \\ p \leq x, q=\left[p^{c}\right]}} \lambda_{\text {sym }^{2} f}(p) \ll \frac{x}{\log ^{A+2} x} .
$$

Theorem 2. Assuming the Riemann Hypothesis of symmetric square L-function is true, (3) holds for all $c \in(0,1)$.

Corollary 1. For almost all $c \in(\varepsilon,(\sqrt{5}+3) / 8-\varepsilon)$, we have

$$
\sum_{\substack{p, q \text { prime } \\ p \leq x, q=\left[p^{c}\right]}} \lambda_{f}^{2}(p)=\frac{x}{c \log ^{2} x}(1+o(1)), x \rightarrow \infty .
$$

Proof. The result follows easily from Theorem 1 and (2), if we notice that $\lambda_{f}^{2}(p)=1+$ $\lambda_{f}\left(p^{2}\right)$.

Corollary 2. Assuming the Riemann Hypothesis of symmetric square L-function is true, (4) holds for all $c \in(0,1)$.

Proof. The result follows from Theorem 2 and (2).

Notation. Throughout the paper, $\varepsilon$ always denotes a sufficiently small positive constant. Let $\delta_{1}(\varepsilon)$ be sufficiently small and depend on $\varepsilon$. We write $f(x) \ll g(x)$, or $f(x)=O(g(x))$, to mean that $|f(x)| \leq C g(x)$. Let $\rho=\sigma+i \eta$ be the nontrivial zero of the symmetric square L-function $L\left(s, \operatorname{sym}^{2} f\right)$. As usual, $\Lambda(n)$ is the von Mangoldt function.

\section{Auxiliary Lemmas}

Lemma 1. Let $\eta$ run through a countable set of reals, with $c(\eta)$ arbitrary complex such that $S(t)=\sum_{\eta} c(\eta) e(\eta t)$ is absolutely convergent. Let $\alpha \in \mathbb{R}, \Theta \in(0,1), \delta=\Theta / \alpha$. Then

$$
\int_{-\alpha}^{\alpha}|S(t)|^{2} d t \ll_{\Theta} \int_{-\infty}^{\infty}\left|\delta^{-1} \sum_{t \leq \eta \leq t+\delta} c(\eta)\right|^{2} d t
$$

Proof. This lemma is Lemma 1 of Gallagher [37].

Lemma 2. Let $T \geq T_{0}$, where $T_{0}$ is a sufficiently large real number. The

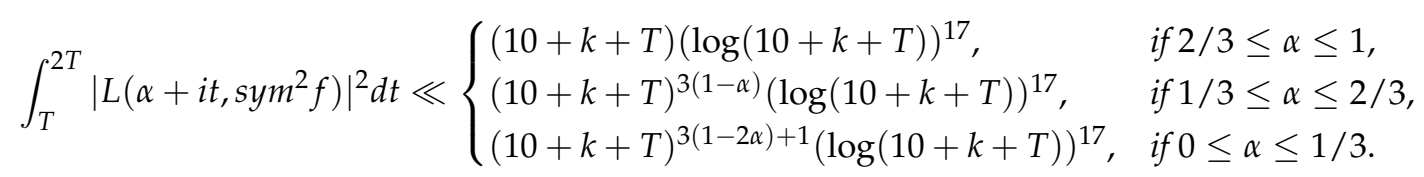

Proof. This lemma is Lemma 3.1 of Lü [33].

Lemma 3. For $1 / 2 \leq \sigma \leq 1, T \geq 3$, define

$$
N(\sigma, T)=\sharp\left\{\varrho=\beta+i \omega: L\left(\varrho, \operatorname{sym}^{2} f\right)=0, \sigma \leq \beta \leq 1,|\omega| \leq T\right\} .
$$


Then we have

$$
N(\sigma, T) \ll T^{\frac{5(1-\sigma)}{3-2 \sigma}+\varepsilon} \text {, if } 1 / 2 \leq \sigma \leq 3 / 4,
$$

and

$$
N(\sigma, T) \ll T^{3(1-\sigma)+\varepsilon}, \text { if } 3 / 4<\sigma \leq 1 .
$$

Proof. From Lemma 2, we have

$$
\int_{T}^{2 T} \mid\left. L\left(1 / 2+i t, \text { sym }^{2} f\right)\right|^{2} d t \ll T^{3 / 2+\varepsilon} .
$$

This combined with Theorem 1.1, 1.2 of Ye and Zhang [38] and [39] gives this lemma.

Lemma 4. For any $u \geq 2, T \geq 2$, we have

$$
\sum_{n<u} \Lambda(n) \lambda_{s y m^{2} f}(n)=-\sum_{\substack{\rho=\sigma+i \eta \\|\eta| \leq T}} \frac{u^{\rho}}{\rho}+O\left(\log ^{3} u+\frac{u \log ^{3}(u T)}{T}\right)
$$

Proof. See, for example, Iwaniec and Kowalski [23]. For convenience of calculation, we reduce the summation range of $n$ from $n \leq u$ to $n<u$. The contribution of $n=u$ is $O\left(\log ^{3} u\right)$, in view of $\left|\lambda_{\text {sym } m^{2} f}\left(p^{h}\right)\right| \leq d_{3}\left(p^{h}\right) \ll \log ^{2} u$.

Lemma 5. Let $L(s, f)$ be an L-function of degree $k$ such that Rankin-Selberg convolutions $L(s, f \otimes$ $f)$ and $L(s, f \otimes \bar{f})$ exist, and the latter has a simple pole at $s=\alpha+i t=1$ while the former is entire if $f \neq \bar{f}$. Suppose that the ramified primes $\left|\alpha_{f}(p)\right| \leq p / 2$. There exists an absolute constant $c>0$ such that $L(s, f)$ has no zeros in the region

$$
\alpha \geq 1-\frac{c}{k^{4} \log (\mathfrak{q}(f)|t|+3)}
$$

where $\mathfrak{q}(f, s)$ is the analytic conductor and $\mathfrak{q}(f)=\mathfrak{q}(f, 0)$.

Proof. This lemma is Theorem 5.10 of Iwaniec and Kowalski [23].

Lemma 6. Let $x, T \in \mathbb{R}^{+}, x \rightarrow \infty$ and

$$
S:=\sum_{\substack{\rho=\sigma+i \eta \\|\eta| \leq T}} x^{\sigma}
$$

we have

$$
S \ll \begin{cases}x^{1 / 2} T^{5 / 4+\varepsilon}, & \text { if } T \geq x^{4 / 5} \\ x^{3 / 2} T^{5 / 2+\varepsilon} e^{-\sqrt{5 \log M \log T},} & \text { if } x^{9 / 20} \leq T<x^{4 / 5} \\ x^{3 / 4} T^{5 / 6+\varepsilon}+x^{1-\beta} T^{3 \beta+\varepsilon}, & \text { if } T<x^{9 / 20}\end{cases}
$$

Proof. Note that

$$
S=\int_{1 / 2}^{1-\beta} x^{\sigma} d_{\sigma} N(\sigma, T)
$$


where $\beta=\beta(T)$ is the width of the zero-free region of the symmetric square $L$-function in Lemma 5. Using integration by parts and Lemma 3, we obtain

$$
\begin{aligned}
S & \ll_{l} x^{1 / 2} T+\int_{1 / 2}^{3 / 4} \exp \left(f_{1}(\sigma)\right) d \sigma+\int_{3 / 4}^{1-\beta} \exp \left(f_{2}(\sigma)\right) d \sigma \\
& :=x^{1 / 2} T+S_{1}+S_{2}
\end{aligned}
$$

where $\ll_{l}$ is fulfilled apart from a fixed number of log-factors,

$$
f_{1}(\sigma)=\frac{5(1-\sigma)}{3-2 \sigma} \log T+\sigma \log x+\varepsilon \log T
$$

and

$$
f_{2}(\sigma)=3(1-\sigma) \log T+\sigma \log x+\varepsilon \log T .
$$

We estimate $S_{1}$ first. The first and second derivatives of $f_{1}(\sigma)$ are

$$
f_{1}^{\prime}(\sigma)=-\frac{5 \log T}{(3-2 \sigma)^{2}}+\log x
$$

and

$$
f_{1}^{\prime \prime}(\sigma)=-\frac{20 \log T}{(3-2 \sigma)^{3}}<0, \text { if } \sigma<3 / 2
$$

Then $f_{1}(\sigma)$ is a concave function as $\sigma<3 / 2$. Let $f_{1}^{\prime}\left(\sigma_{1}\right)=0$, we have $\sigma_{1}=\frac{3}{2}-\frac{1}{2} \sqrt{\frac{5 \log T}{\log x}}$. So we have the following three cases.

Case 1. When $T \geq x^{4 / 5}$. In this case, we have $\sigma_{1} \leq 1 / 2$ and $f_{1}(\sigma)$ is monotonically decreasing in $[1 / 2,3 / 4]$. Hence

$$
\max _{\sigma \in[1 / 2,3 / 4]} f_{1}(\sigma)=f_{1}(1 / 2)=(5 \log T) / 4+(\log x) / 2+\varepsilon \log T .
$$

Case 2. When $x^{9 / 20} \leq T<x^{4 / 5}$. In this case, the function $f_{1}(\sigma)$ takes the extreme value at $\sigma_{1}$, which gives

$$
\max _{\sigma \in[1 / 2,3 / 4]} f_{1}(\sigma)=f_{1}\left(\sigma_{1}\right)=(5 \log T) / 2+(3 \log x) / 2-\sqrt{5 \log T \log x}+\varepsilon \log T .
$$

Case 3. When $T<x^{9 / 20}$. In this case, we have $\sigma_{1}>3 / 4$ and $f_{1}(\sigma)$ is monotonically increasing in $[1 / 2,3 / 4]$. Hence,

$$
\max _{\sigma \in[1 / 2,3 / 4]} f_{1}(\sigma)=f_{1}(3 / 4)=(5 \log T) / 6+(3 \log x) / 4+\varepsilon \log T .
$$

Combining all the above cases, we have

$$
S_{1}=\int_{\frac{1}{2}}^{\frac{3}{4}} \exp \left(f_{1}(\sigma)\right) d \sigma \ll \begin{cases}x^{1 / 2} T^{5 / 4+\varepsilon}, & \text { if } T \geq x^{4 / 5} \\ x^{3 / 2} T^{5 / 2+\varepsilon} e^{-\sqrt{5 \log x \log T},}, & \text { if } x^{9 / 20} \leq T<x^{4 / 5} \\ x^{3 / 4} T^{5 / 6+\varepsilon}, & \text { if } T<x^{9 / 20}\end{cases}
$$

Next, we need to estimate $S_{2}$. It is easy to see that $f_{2}(\sigma)$ is linear function in $[3 / 4,1-\beta]$, hence

$$
\begin{aligned}
f_{2}(\sigma) & \ll \max \left(f_{2}(3 / 4), f_{2}(1-\beta)\right) \\
& =\max ((3 \log T) / 4+(3 \log x) / 4,3 \beta \log T+(1-\beta) \log x)+\varepsilon \log T .
\end{aligned}
$$


From (6), (7) and (8), we obtain

$$
S \ll_{l} \begin{cases}x^{1 / 2} T+x^{1 / 2} T^{5 / 4+\varepsilon}+x^{3 / 4} T^{3 / 4+\varepsilon}, & \text { if } T \geq x^{4 / 5} ; \\ x^{1 / 2} T+x^{3 / 2} T^{5 / 2+\varepsilon} e^{-\sqrt{5 \log x \log T}}+x^{3 / 4} T^{3 / 4+\varepsilon}, & \text { if } x^{9 / 20} \leq T<x^{4 / 5} \\ x^{1 / 2} T+x^{3 / 4} T^{5 / 6+\varepsilon}+x^{1-\beta} T^{3 \beta+\varepsilon}, & \text { if } T<x^{9 / 20},\end{cases}
$$

which proves this lemma.

Lemma 7. Let $x_{0}:=2, x_{v+1}:=x_{v}+x_{v} \log ^{-2} x_{v}$. Let $\gamma_{0}$ be a constant and

$$
D\left(\gamma_{0}, x_{v}, T\right)=\int_{\gamma_{0}}^{\gamma_{0}+\log ^{-2} x_{v} \mid}\left|\sum_{x_{v}^{1 / \gamma}<m \leq x_{v+1}^{1 / \gamma}} \Lambda(m) \sum_{\substack{\rho=\sigma+i \eta \\ T / 2<|\eta| \leq T}} \frac{(m+1)^{\gamma \rho}-m^{\gamma \rho}}{\rho}\right|^{2} d \gamma
$$

If $T>x_{v}^{1 / \gamma_{0}-\delta}$, we have

$$
D\left(\gamma_{0}, x_{v}, T\right) \ll x_{v}^{2 / \gamma_{0}} T^{-3}\left(\sum_{\substack{\rho=\sigma+i \eta \\ T / 2<|\eta| \leq T}} x_{v}^{\sigma}\right)^{2} .
$$

If $T \leq x_{v}^{1 / \gamma_{0}-\delta}$, we have

$$
D\left(\gamma_{0}, x_{v}, T\right) \ll x_{v}^{2 / \gamma_{0}} T^{-3}\left(\sum_{\substack{\rho=\sigma+i \eta \\ T / 2<|\eta| \leq T}} x_{v}^{\sigma}\right)^{2}\left(T x_{v}^{-1 / \gamma_{0}}\right)^{2} .
$$

Proof. This lemma follows from (13) and (16) of Dufner [26].

\section{Proof of Theorem 2}

In this section, we write $\gamma=1 / c$. Then we have

$$
\Pi_{2, c}(x):=\sum_{\substack{p, q p p r i m e \\ p \leq x, q=\left[p^{c}\right]}} \lambda_{s y m^{2} f}(p)=\sum_{\substack{p, q \text { prime } \\ p \leq x, q \leq p^{c}<(q+1)}} \lambda_{s y m^{2} f}(p)=\sum_{q \leq x^{c}} \sum_{\substack{p \leq x \\ q^{\gamma} \leq p<(q+1)^{\gamma}}} \lambda_{\text {sym }^{2} f}(p) .
$$

We split the summation range of $q$ into two parts: $q \leq x^{c}-1$ and $x^{c}-1<q \leq x^{c}$ to get

$$
\begin{aligned}
\Pi_{2, c}(x) & =\sum_{q \leq x^{c}-1} \sum_{q^{\gamma} \leq p<(q+1)^{\gamma}} \lambda_{s y m^{2} f}(p)+\sum_{x^{c}-1<q \leq x^{c}} \sum_{q^{\gamma} \leq p \leq x} \lambda_{s y m^{2} f}(p) \\
& =\sum_{q \leq x^{c}} \sum_{q^{\gamma} \leq p<(q+1)^{\gamma}} \lambda_{s y m^{2} f}(p)-\sum_{x^{c}-1<q \leq x^{c}} \sum_{x<p<(q+1)^{\gamma}} \lambda_{\text {sym }^{2} f}(p) \\
& :=E_{1}-E_{2} .
\end{aligned}
$$

For $E_{2}$, there is at most one prime $q_{x}$ satisfying $x^{c}-1<q_{x} \leq x^{c}$, hence

$$
E_{2} \ll \sum_{q_{x}^{\gamma}<p \leq\left(q_{x}+1\right)^{\gamma}}\left|\lambda_{s y m^{2} f}(p)\right| \ll\left(q_{x}+1\right)^{\gamma}-q_{x}^{\gamma} \ll x^{1-c},
$$

if we notice that $\left|\lambda_{\text {symm }^{2} f}(p)\right| \leq d_{3}(p) \ll 1$.

For $E_{1}$, by the definition of the von Mangoldt function, we have

$$
E_{1}=\sum_{q \leq x^{c}} \sum_{q^{\gamma} \leq n<(q+1)^{\gamma}} \frac{\Lambda(n) \lambda_{\text {sym }^{2} f}(n)}{\log n}+O\left(\sum_{\substack{q \leq x^{c} \\ q^{\gamma} \leq n=p^{h}<(q+1)^{\gamma} \\ h \geq 2}} \frac{\lambda_{s y m^{2} f}\left(p^{h}\right) \log p}{\log p^{h}}\right) .
$$


The error term of the above formula contributes

$$
\begin{aligned}
& \ll \sum_{q \leq x^{c} q_{q^{\gamma} \leq p^{h}<(q+1)^{\gamma}}}\left|\lambda_{\text {sym }^{2} f}\left(p^{h}\right)\right| \\
& =\sum_{q \leq x^{c}} \sum_{q^{\gamma} \leq p^{2}<(q+1)^{\gamma}}\left|\lambda_{s y m^{2} f}\left(p^{2}\right)\right|+\sum_{q \leq x^{c}} \sum_{q^{\gamma} \leq p^{h}<(q+1)^{\gamma}}\left|\lambda_{s y m^{2} f}\left(p^{h}\right)\right|
\end{aligned}
$$

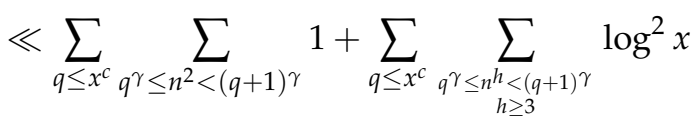

$$
\begin{aligned}
& \ll \sum_{q \leq x^{c}}\left((q+1)^{\gamma / 2}-q^{\gamma / 2}\right)+\sum_{q \leq x^{c}}\left((q+1)^{\gamma / 3}-q^{\gamma / 3}\right) \log ^{3} x \\
& \ll \sum_{q \leq x^{c}} q^{\gamma / 2-1}+\sum_{q \leq x^{c}} q^{\gamma / 3-1} \log ^{3} x \ll x^{1 / 2} .
\end{aligned}
$$

We use the same method to deal with the main term of (13) and get

$$
E_{1}=\sum_{2 \leq m \leq x^{c}} \frac{\Lambda(m)}{\log m} \sum_{m^{\gamma} \leq n<(m+1)^{\gamma}} \frac{\Lambda(n) \lambda_{\text {sym }^{2} f}(n)}{\log n}+O\left(x^{1-c / 2}\right)+O\left(x^{1 / 2}\right),
$$

where the first $O$-term comes from

$$
\begin{aligned}
& \sum_{\substack{m=h^{h} \leq x^{c} \\
h \geq 2}} \frac{\Lambda(m)}{\log m} \sum_{m^{\gamma} \leq n<(m+1)^{\gamma}} \frac{\Lambda(n) \lambda_{\text {symm }^{2} f}(n)}{\log n} \\
& =\sum_{\substack{m=h^{h} \leq x^{c} \\
h>2}} \frac{\log q}{\log q^{h}} \sum_{m \gamma \leq p^{r}<(m+1)^{\gamma}} \frac{\lambda_{s y m^{2} f}\left(p^{r}\right) \log p}{\log p^{r}} \\
& \ll \sum_{\substack{m=q^{h} \leq x^{c} \\
h \geq 2}} \sum_{m^{\gamma} \leq p^{r}<(m+1)^{\gamma}}\left|\lambda_{s y m^{2} f}\left(p^{r}\right)\right| \ll x^{1-c / 2} .
\end{aligned}
$$

Let

$$
W(x):=\sum_{2 \leq m \leq x^{c}} \frac{\Lambda(m)}{\log m} \sum_{m^{\gamma} \leq n<(m+1)^{\gamma}} \frac{\Lambda(n) \lambda_{\text {sym }^{2} f}(n)}{\log n} .
$$

By the range of $n$ and Taylor's formula, we have

$$
\log n=\gamma \log m+O\left(m^{-1}\right)
$$

and

$$
\frac{1}{\log n}=\frac{1}{\gamma \log m}+O\left(\frac{1}{m \log ^{2} m}\right)
$$

Then

$$
W(x)=\gamma^{-1} \sum_{2 \leq m \leq x} \frac{\Lambda(m)}{\log ^{2} m} \sum_{m^{\gamma} \leq n<(m+1)^{\gamma}} \Lambda(n) \lambda_{s y m^{2} f}(n)+O\left(x^{1-c} \log ^{3} x\right),
$$


where the $O$-term comes from

$$
\begin{aligned}
& \sum_{2 \leq m \leq x^{c}} \frac{\Lambda(m)}{m \log ^{3} m} \sum_{m \gamma \leq n<(m+1)^{\gamma}} \Lambda(n) \lambda_{s y m^{2} f}(n) \\
\ll & \log ^{3} x \sum_{2 \leq m \leq x^{c}} m^{\gamma-2} \log ^{-3} m \\
\ll & x^{1-c} \log ^{3} x \sum_{2 \leq m \leq x^{c}} \frac{1}{m \log ^{3} m} \\
\ll & x^{1-c} \log ^{3} x .
\end{aligned}
$$

From (14), (15) and partial summation, we obtain

$$
E_{1} \ll \log ^{-2} x \cdot \max _{x_{0} \leq x}\left|\Psi_{2, c}\left(x_{0}\right)\right|+x^{1-c} \log ^{3} x+x^{1-c / 2}+x^{1 / 2},
$$

where

$$
\Psi_{2, c}(x)=\sum_{m \leq x^{c}} \Lambda(m) \sum_{m \gamma \leq n<(m+1)^{\gamma}} \Lambda(n) \lambda_{\text {sym }^{2} f}(n) .
$$

To get (3), it suffices to prove that

$$
\left|\Psi_{2, c}(x)\right| \ll x \log ^{-A} x, \text { for any } A>0 .
$$

The inequality (17) will be proved from a variant of (17) for short intervals. Let $x_{0}:=2$, $x_{v+1}:=x_{v}+x_{v} \log ^{-2} x_{v}$, we can see that the inequality

$$
\left|\Delta \Psi_{2, c}\left(x_{v}\right)\right|=\left|\sum_{x_{v}^{c}<m \leq x_{v+1}^{c}} \Lambda(m) \sum_{m^{\gamma} \leq n<(m+1)^{\gamma}} \Lambda(n) \lambda_{s y m^{2} f}(n)\right| \ll x_{v} \log ^{-A-2} x_{v}
$$

implies (17). We use Lemma 4 and get

$$
\sum_{m^{\gamma} \leq n<(m+1)^{\gamma}} \Lambda(n) \lambda_{\text {sym }^{2} f}(n)=-\sum_{\substack{\rho=\sigma+i \eta \\|\eta| \leq T_{v}}} \frac{(m+1)^{\gamma \rho}-m^{\gamma \rho}}{\rho}+O\left(\log ^{3} m+\frac{(m+1)^{\gamma} \log ^{3}(m T)}{T}\right) .
$$

Taking $T_{v}=x_{v} \log ^{-2 A-3} x_{v}$, then we obtain

$$
\left|\Delta \Psi_{2, c}\left(x_{v}\right)\right| \ll\left|\Phi\left(\gamma, x_{v}, T_{v}\right)\right|+x_{v}^{c} \log ^{2 A+4} x_{v},
$$

where

$$
\Phi\left(\gamma, x_{v}, T_{v}\right)=\sum_{x_{v}^{c}<m \leq x_{v+1}^{c}} \Lambda(m) \sum_{\substack{\rho=\sigma+i \eta \\|\eta| \leq T_{v}}} \frac{(m+1)^{\gamma \rho}-m^{\gamma \rho}}{\rho} .
$$

Under the Riemann Hypothesis, we have

$$
\begin{aligned}
\Phi\left(\gamma, x_{v}, T_{v}\right) & =\sum_{x_{v}^{c}<m \leq x_{v+1}^{c}} \Lambda(m) \int_{m \gamma}^{(m+1)^{\gamma}} \sum_{\substack{\rho=\sigma+i \eta \\
|\eta| \leq T_{v}}} t^{-1 / 2+i \eta} d t \\
& =\sum_{x_{v}^{c}<m \leq x_{v+1}^{c}} \Lambda(m) \int_{m \gamma}^{(m+1)^{\gamma}} \sum_{\substack{\rho=\sigma+i \eta \\
|\eta| \leq T_{v}}} e^{(-1 / 2+i \eta) \log t} d t .
\end{aligned}
$$


Making the change of variables $u=\log t$, we deduce that

$$
\Phi\left(\gamma, x_{v}, T_{v}\right)=\sum_{x_{v}^{c}<m \leq x_{v+1}^{c}} \Lambda(m) \int_{\gamma \log m}^{\gamma \log (m+1)} e^{u / 2} \sum_{\substack{\rho=\sigma+i \eta \\|\eta| \leq T_{v}}} e^{i \eta u} d u .
$$

Using the Cauchy-Schwarz inequality twice, we get

$$
\begin{aligned}
\left|\Phi\left(\gamma, x_{v}, T_{v}\right)\right|^{2} & \ll \sum_{x_{v}^{c}<m \leq x_{v+1}^{c}} \Lambda^{2}(m) \sum_{x_{v}^{c}<m \leq x_{v+1}^{c}}\left|\int_{\gamma \log m}^{\gamma \log (m+1)} e^{u / 2} \sum_{\substack{\rho=\sigma+i \eta \\
|\eta| \leq T_{v}}} e^{i \eta u} d u\right|^{2} \\
& \ll \sum_{x_{v}^{c}<m \leq x_{v+1}^{c}} \Lambda^{2}(m) \sum_{x_{v}^{c}<m \leq x_{v+1}^{c}} m^{\gamma-1} \int_{\gamma \log m}^{\gamma \log (m+1) \mid}\left|\sum_{\substack{\rho=\sigma+i \eta \\
|\eta| \leq T_{v}}} e^{i \eta u}\right|^{2} d u \\
& \ll x_{v} \log ^{-1} x_{v} \cdot \int_{\log x_{v}}^{\gamma+\log x_{v+1} \mid}\left|\sum_{\substack{\rho=\sigma+i \eta \\
|\eta| \leq T_{v}}} e^{i \eta \eta}\right|^{2} d u .
\end{aligned}
$$

Making the change of variables $t=u(2 \pi)^{-1}-\left(\gamma+\log x_{v+1}+\log x_{v}\right)(4 \pi)^{-1}$, we deduce that the last integral in (22) can be written as

$$
2 \pi \int_{-\alpha}^{\alpha}\left|\sum_{|\eta| \leq T_{v}} c(\eta) e(\eta t)\right|^{2} d t
$$

where $\alpha=\left(\gamma+\log x_{v+1}-\log x_{v}\right)(4 \pi)^{-1}$ and

$$
c(\eta)= \begin{cases}e^{i \eta\left(\gamma+\log x_{v+1}+\log x_{v}\right) / 2}, & \text { if }|\eta| \leq T_{v} \\ 0, & \text { otherwise }\end{cases}
$$

Applying Lemma 1 with $\Theta=1 / 2$ and $\delta=\Theta / \alpha \sim 2 \pi / \gamma$ to estimate the integral in (23), we have

$$
\begin{aligned}
& \ll \int_{-\infty}^{\infty}\left|\delta^{-1} \sum_{\substack{t \leq \eta \leq t+\delta \\
|\eta| \leq T_{v}}} c(\eta)\right|^{2} d t \\
& =\int_{-\infty}^{\infty} \delta^{-2} \sum_{\substack{t \leq \eta_{1}, \eta_{2} \leq t+\delta \\
\left|\eta_{1},\right| \eta_{2} \mid \leq T_{v}}} e^{i\left(\eta_{1}-\eta_{2}\right)\left(\gamma+\log x_{v+1}+\log x_{v}\right) / 2} d t \\
& \ll \delta^{-2} \cdot \delta^{2} \cdot T_{v} \ll x_{v} \log ^{-2 A-3} x_{v} .
\end{aligned}
$$

From (20), (22) and (24), we get

$$
\left|\Delta \Psi_{2, c}\left(x_{v}\right)\right| \ll x_{v} \log ^{-A-2} x_{v}+x_{v}^{c} \log ^{2 A+4} x_{v} .
$$

This combined with (16) and (17) gives

$$
E_{1} \ll x \log ^{-A-2} x .
$$

Theorem 2 follows from (11), (12) and (25). 


\section{Proof of Theorem 1}

To prove Theorem 1, we need Lemma 6 and Lemma 7. Furthermore, we only need to estimate $\Phi\left(\gamma, x_{v}, T_{v}\right)$ unconditionally. Note that

$$
\Phi\left(\gamma, x_{v}, T_{v}\right) \ll \log x_{v} \cdot \max _{1 \ll T \leq T_{v}}\left|\Phi_{1}\left(\gamma, x_{v}, T\right)\right|,
$$

where

$$
\Phi_{1}\left(\gamma, x_{v}, T\right)=\sum_{x_{v}^{c}<m \leq x_{v+1}^{c}} \Lambda(m) \sum_{\substack{\rho=\sigma+i \eta \\ T / 2<|\eta| \leq T}} \frac{(m+1)^{\rho \gamma}-m^{\rho \gamma}}{\rho} .
$$

We consider the following integral mean value of $\Phi_{1}\left(\gamma, x_{v}, T\right)$,

$$
D\left(\gamma_{0}, x_{v}, T\right)=\int_{\gamma_{0}}^{\gamma_{0}+\log ^{-2} x_{v}}\left|\Phi_{1}\left(\gamma, x_{v}, T\right)\right|^{2} d \gamma
$$

with $\gamma_{0} \in\left[\frac{1}{(\sqrt{5}+3) / 8-\varepsilon}, \frac{1}{\varepsilon}\right]$.

From Lemma 6, we know that the upper bound of $S$ depends on the range of $T$, so we have the following three cases.

Case 1. When $T \geq x_{v}^{4 / 5}$.

In this case, we have $x_{v}^{1 / \gamma_{0}-\delta_{1}(\varepsilon)}<T$ and use (9) to get

$$
\begin{aligned}
D\left(\gamma_{0}, x_{v}, T_{v}\right) & \ll x_{v}^{2 / \gamma_{0}} T^{-3}\left(x_{v}^{1 / 2} T^{5 / 4+\varepsilon}\right)^{2}=x_{v}^{1+2 / \gamma_{0}} T^{-1 / 2+2 \varepsilon} \\
& \ll x_{v}^{1+3 / 2 \gamma_{0}+2 \varepsilon / \gamma_{0}+(1 / 2-2 \varepsilon) \delta_{1}(\varepsilon)} .
\end{aligned}
$$

Note that if $1 / \gamma_{0} \leq(\sqrt{5}+3) / 8-\varepsilon$, we have

$$
D\left(\gamma_{0}, x_{v}, T\right) \ll x_{v}^{2-\varepsilon} .
$$

Case 2. When $x_{v}^{9 / 20} \leq T<x_{v}^{4 / 5}$.

In case of $x_{v}^{1 / \gamma_{0}-\delta_{1}(\varepsilon)}<T$, we use (9) and get

$$
\begin{aligned}
D\left(\gamma_{0}, x_{v}, T_{v}\right) & \ll x_{v}^{2 / \gamma_{0}} T^{-3}\left(x_{v}^{3 / 2} T^{5 / 2+\varepsilon} e^{-\sqrt{5 \log x_{v} \log T}}\right)^{2} \\
& =x_{v}^{3+2 / \gamma_{0}} T^{2+2 \varepsilon} e^{-2} \sqrt{5 \log x_{v} \log T} \\
& =e^{\left[(2+2 \varepsilon)\left(\sqrt{\frac{\log T}{\log x_{v}}}\right)^{2}-2 \sqrt{5 \frac{\log T}{\log x_{v}}}+3+\frac{2}{\gamma_{0}}\right] \log x_{v}} \\
& =x_{v}^{(2+2 \varepsilon)\left(\sqrt{\frac{\log T}{\log x_{v}}}\right)^{2}-2 \sqrt{5 \frac{\log T}{\log x_{v}}}+3+2 / \gamma_{0}},
\end{aligned}
$$

For convenience, we take $t=\sqrt{\frac{\log T}{\log x_{v}}}$ and consider a quadratic function $g(t)=$ $(2+2 \varepsilon) t^{2}-2 \sqrt{5} t+3+2 / \gamma_{0}$.

If $x_{v}^{1 / \gamma_{0}-\delta_{1}(\varepsilon)} \leq x_{v}^{9 / 20} \leq T$, we have $t \in[\sqrt{9 / 20}, \sqrt{4 / 5})$ and $g(t)$ is monotonically decreasing in this interval. Hence,

$$
g(t) \leq g(\sqrt{9 / 20})=9(1+\varepsilon) / 10+2 / \gamma_{0} \leq 9 / 5+9 / 10 \varepsilon+2 \delta_{1}(\varepsilon)
$$

with $1 / \gamma_{0} \leq 9 / 20+\delta_{1}(\varepsilon)$. Therefore,

$$
D\left(\gamma_{0}, x_{v}, T\right) \ll x_{v}^{2-\varepsilon} .
$$


and

$$
\text { If } x_{v}^{9 / 20}<x_{v}^{1 / \gamma_{0}-\delta_{1}(\varepsilon)}<T<x_{v}^{4 / 5} \text {, we get analogously } t \in\left(\sqrt{1 / \gamma_{0}-\delta_{1}(\varepsilon)}, \sqrt{4 / 5}\right)
$$

$$
\begin{aligned}
g(t) & <g\left(\sqrt{1 / \gamma_{0}-\delta_{1}(\varepsilon)}\right)=3+4 / \gamma_{0}-2 \sqrt{5\left(1 / \gamma_{0}-\delta_{1}(\varepsilon)\right)}+2 \varepsilon / \gamma_{0}-(2+2 \varepsilon) \delta_{1}(\varepsilon) \\
& \leq 3+4 / \gamma_{0}-2 \sqrt{5} / \sqrt{\gamma_{0}}+2 \varepsilon / \gamma_{0}+\left(\sqrt{10 \gamma_{0}}-2-2 \varepsilon\right) \delta_{1}(\varepsilon) \\
& \leq 2-\varepsilon
\end{aligned}
$$

where we choose $\delta_{1}(\varepsilon)$ sufficiently small and use the elementary inequality

$$
\sqrt{1-x} \geq 1-x / \sqrt{2}, \text { for } x \in[0,1 / 2] .
$$

Therefore,

$$
D\left(\gamma_{0}, x_{v}, T\right) \ll x_{v}^{2-\varepsilon} .
$$

In case of $T \leq x_{v}^{1 / \gamma_{0}-\delta_{1}(\varepsilon)}$, we use (10) and get

$$
\begin{aligned}
D\left(\gamma_{0}, x_{v}, T\right) & \ll x_{v}^{3+2 / \gamma_{0}} T^{2+2 \varepsilon} e^{-2 \sqrt{5 \log T \log x_{v}}}\left(T x_{v}^{-1 / \gamma_{0}}\right)^{2} \\
& =x_{v}^{3} T^{4+2 \varepsilon} e^{-2 \sqrt{5 \log T \log x_{v}}} \\
& =x_{v}^{(4+2 \varepsilon)\left(\sqrt{\frac{\log T}{\log x_{v}}}\right)^{2}-2 \sqrt{5 \frac{\log T}{\log x_{v}}}+3},
\end{aligned}
$$

so we consider a new quadratic function $g_{1}(t)=(4+2 \varepsilon) t^{2}-2 \sqrt{5} t+3$.

If $x_{v}^{9 / 20} \leq T \leq x_{v}^{1 / \gamma_{0}-\delta_{1}(\varepsilon)}<x_{v}^{4 / 5}$, we get $t \in\left[\sqrt{9 / 20}, \sqrt{1 / \gamma_{0}-\delta_{1}(\varepsilon)}\right]$ and

$$
\begin{aligned}
g_{1}(t) & \leq g_{1}\left(1 / \gamma_{0}-\delta_{1}(\varepsilon)\right)=4\left(1 / \gamma_{0}-\delta_{1}(\varepsilon)\right)-2 \sqrt{5} \cdot \sqrt{1 / \gamma_{0}-\delta_{1}(\varepsilon)}+3+2 \varepsilon / \gamma_{0}-2 \varepsilon \delta_{1}(\varepsilon) \\
& \leq 4 / \gamma_{0}-2 \sqrt{5} / \sqrt{\gamma_{0}}+3+2 \varepsilon / \gamma_{0}+\left(\sqrt{10 \gamma_{0}}-4-2 \varepsilon\right) \delta_{1}(\varepsilon) \\
& \leq 2-\varepsilon .
\end{aligned}
$$

Therefore,

$$
D\left(\gamma_{0}, x_{v}, T\right) \ll x_{v}^{2-\varepsilon} .
$$

Case 3. When $T<x_{v}^{9 / 20}$.

$$
\text { If } x_{v}^{1 / \gamma_{0}-\delta_{1}(\varepsilon)}<T<x_{v}^{9 / 20} \text {, we use (9) to get }
$$

$$
x_{v}^{2 / \gamma_{0}} T^{-3}\left(x_{v}^{3 / 4} T^{5 / 6+\varepsilon}\right)^{2}=x_{v}^{3 / 2+2 / \gamma_{0}} T^{-4 / 3+2 \varepsilon} \ll x_{v}^{2-\varepsilon}
$$

and

$$
x_{v}^{2 / \gamma_{0}} T^{-3}\left(x_{v}^{1-\beta} T^{3 \beta+\varepsilon}\right)^{2}=x_{v}^{2-2 \beta+2 / \gamma_{0}} T^{-3+6 \beta+2 \varepsilon} \ll x_{v}^{2-\varepsilon} .
$$

Therefore,

$$
\begin{array}{r}
D\left(\gamma_{0}, x_{v}, T\right) \ll x_{v}^{2 / \gamma_{0}} T^{-3}\left(x_{v}^{3 / 4} T^{5 / 6+\varepsilon}+x_{v}^{1-\beta} T^{3 \beta+\varepsilon}\right)^{2} \ll x_{v}^{2-\varepsilon} . \\
\text { If } T \leq x_{v}^{1 / \gamma_{0}-\delta_{1}(\varepsilon)}<x_{v}^{9 / 20} \text { or } T<x_{v}^{9 / 20} \leq x_{v}^{1 / \gamma_{0}-\delta_{1}(\varepsilon)}, \text { we use (10) to get } \\
x_{v}^{2 / \gamma_{0}} T^{-3}\left(x_{v}^{3 / 4} T^{5 / 6+\varepsilon}\right)^{2}\left(T x_{v}^{-1 / \gamma_{0}}\right)^{2} \ll x_{v}^{3 / 2} T^{2 / 3+2 \varepsilon} \ll x_{v}^{2-\varepsilon}
\end{array}
$$

and

$$
x_{v}^{2 / \gamma_{0}} T^{-3}\left(x_{v}^{1-\beta} T^{3 \beta+\varepsilon}\right)^{2}\left(T x_{v}^{-1 / \gamma_{0}}\right)^{2} \ll x_{v}^{2-2 \beta} T^{-1+6 \beta+2 \varepsilon} \ll x_{v}^{2-\varepsilon}
$$


Therefore,

$$
D\left(\gamma_{0}, x_{v}, T\right) \ll x_{v}^{2 / \gamma_{0}} T^{-3}\left(x_{v}^{3 / 4} T^{5 / 6+\varepsilon}+x_{v}^{1-\beta} T^{3 \beta+\varepsilon}\right)^{2}\left(T x_{v}^{-1 / \gamma_{0}}\right)^{2} \ll x_{v}^{2-\varepsilon} .
$$

Combining all the above cases, we obtain

$$
D\left(\gamma_{0}, x_{v}, T\right)=\int_{\gamma_{0}}^{\gamma_{0}+\log ^{-2} x_{v}}\left|\Phi_{1}\left(\gamma, x_{v}, T\right)\right|^{2} d \gamma \ll x_{v}^{2-\varepsilon} .
$$

Inequality (20) gives us

$$
\begin{aligned}
\int_{\gamma_{0}}^{\gamma_{0}+\log ^{-2} x_{v}}\left|\Psi_{2, c}(x)\right|^{2} d \gamma & \ll \log ^{4} x \cdot \int_{\gamma_{0}}^{\gamma_{0}+\log ^{-2} x_{v}}\left|\Delta \Psi_{2, c}\left(x_{v}\right)\right|^{2} d \gamma \\
& \ll \log ^{4} x \cdot \int_{\gamma_{0}}^{\gamma_{0}+\log ^{-2} x_{v}}\left|\Phi\left(\gamma, x_{v}, T_{v}\right)\right|^{2} d \gamma+x_{v}^{2 c} \log ^{4 A+10} x \\
& \ll \log ^{6} x \cdot \max _{1 \ll T \leq T_{v}} \int_{\gamma_{0}}^{\gamma_{0}+\log ^{-2} x_{v}}\left|\Phi_{1}\left(\gamma, x_{v}, T\right)\right|^{2} d \gamma+x_{v}^{2 c} \log ^{4 A+10} x \\
& \ll x^{2-\varepsilon} .
\end{aligned}
$$

Let $\gamma_{0}:=\frac{1}{(\sqrt{5}+3) / 8-\varepsilon^{\prime}}, \gamma_{i+1}:=\gamma_{i}+\frac{1}{\log ^{2} x_{v}}$ and $\mathcal{A}_{i}:=\left\{\gamma \in\left[\gamma_{i}, \gamma_{i+1}\right] /\left|\Psi_{2, c}(x)\right|>\right.$ $\left.x \log ^{-A} x\right\}$. Then by Tschebytschev's inequality, we obtain

$$
\begin{aligned}
\mu\left(\mathcal{A}_{i}\right) & \ll x^{-2} \log ^{2 A} x \cdot \int_{\gamma_{i}}^{\gamma_{i+1}}\left|\Psi_{2, c}(x)\right|^{2} d \gamma \\
& \ll x^{-\varepsilon} \log ^{2 A} x \ll x^{-\varepsilon} .
\end{aligned}
$$

Author Contributions: Conceptualization, X.H. and X.Y.; Methodology, D.Z.; Software, X.H.; Validation, X.H., X.Y. and D.Z.; Formal Analysis, X.H.; Investigation, X.H.; Resources, X.Y.; Data Curation, D.Z.; Writing—Original Draft Preparation, X.H.; Writing—Review \& Editing, X.Y.; Visualization, D.Z.; Supervision, D.Z.; Project Administration, D.Z.; Funding Acquisition, X.Y. and D.Z. All authors have read and agreed to the published version of the manuscript.

Funding: This research was funded by National Natural Science Foundation of China (Grant No. 11771256 and No. 11801327), and Natural Science Foundation of Shandong Province (Grant No. ZR201709280100).

Institutional Review Board Statement: Not applicable.

Informed Consent Statement: Not applicable.

Data Availability Statement: Not applicable.

Acknowledgments: The author would like to thank the referees for their many useful comments.

Conflicts of Interest: The authors declare no conflict of interest.

\section{References}

1. Deligne, P. La conjecture de Weil. I. Inst. Hautes Etudes Sci. Publ. Math. 1974, 43, 273-307. [CrossRef]

2. Deligne, P. La conjecture de Weil. II. Inst. Hautes Etudes Sci. Publ. Math. 1980, 52, 137-252. [CrossRef]

3. Chandrasekharan, K.; Narasimhan, R. Functional equations with multiple gamma factors and the average order of arithmetical functions. Ann. Math. 1962, 76, 93-136. [CrossRef]

4. $\quad$ Davenport, H. On certain exponential sums. J. Reine Angew. Math. 1933, 169, 158-176.

5. Hafner, J.L.; Ivić, A. On sums of Fourier coefficients of cusp forms. Enseign. Math. 1989, 35, 373-382.

6. Hecke, E. Theorie der Eisensteinsche Reihen höherer Stufe und ihre Anwendung auf Funktionentheorie und Arithmetik. Abh. Math. Sem. Univ. Hamburg 1927, 5, 199-224. [CrossRef]

7. Jiang, Y.J.; Lü, G.S.; Yan, X.F. Mean value theorem connected with Fourier coefficients of Hecke-Maass forms for $S L(m, \mathbb{Z})$. Math. Proc. Camb. Philos. Soc. 2016, 161, 339-356. [CrossRef] 
8. Kloosterman, H.D. Asymptotische Formeln für die Fourier-koeffizienten ganzer Modulformen. In Abhandlungen aus dem Mathematischen Seminar der Universität Hamburg; Springer: Berlin/Heidelberg, Germany, 1927; Volume 5, pp. $337-353$.

9. Lao, H.X. The cancellation of Fourier coefficients of cusp forms over different sparse sequences. Acta Math. Sin. (Engl. Ser.) 2013, 29, 1963-1972. [CrossRef]

10. Lao, H.X. On comparing Hecke eigenvalues of cusp forms. Acta Math. Hungar. 2020, 160, 58-71. [CrossRef]

11. Liu, H.F.; Li, S.; Zhang, D.Y. Power moments of automorphic L-function attached to Maass forms. Int. J. Number Theory 2016, 12, 427-443. [CrossRef]

12. Liu, H.F.; Zhang, R. Some problems involving Hecke eigenvalues. Acta Math. Hungar. 2019, 159, 287-298. [CrossRef]

13. Rankin, R.A. Sums of Cusp Form Coefficients; Automorphic forms and analytic number theory (Montreal, PQ, 1989); University Montreal: Montreal, QC, Canada, 1990; pp. 115-121.

14. Saljé, H. Zur Abschätzung der Fourierkoeffizienten ganzer Modulformen. Math. Z. 1933, 36, $263-278$.

15. Song, P.; Zhai, W.G.; Zhang, D.Y. Power moments of Hecke eigenvalues for congruence group. J. Number Theory 2019, 198, 139-158. [CrossRef]

16. Walfisz, A. Uber die Koeffizientensummen einiger Moduformen. Math. Ann. 1933, 108, 75-90. [CrossRef]

17. Weil, A. On some exponential sums. Proc. Nat. Acad. Sci. USA 1948, 34, 204-207. [CrossRef]

18. Wilton, J.R. A note on Ramanujans arithmetical function $\tau(n)$. Proc. Cambridge Philos. Soc. 1929, 25, 121-129. [CrossRef]

19. Wu, J. Power sums of Hecke eigenvalues and applications. Acta Arith. 2009, 137, 333-344. [CrossRef]

20. Zhang, D.Y.; Lau, Y-K.; Wang, Y.N. Remark on the paper “On products of Fourier coefficients of cusp forms”. Arch. Math. (Basel) 2017, 108, 263-269. [CrossRef]

21. Zhang, D.Y.; Wang, Y.N. Higher-power moments of Fourier coefficients of holomorphic cusp forms for the congruence subgroup $\Gamma_{0}(N)$. Ramanujan J. 2018, 47, 685-700. [CrossRef]

22. Zhang, D.Y.; Wang, Y.N. Ternary quadratic form with prime variables attached to Fourier coefficients of primitive holomorphic cusp form. J. Number Theory 2017, 176, 211-225. [CrossRef]

23. Iwaniec, H.; Kowalski, E. Analytic Number THeory; American Mathematical Society Colloquium Publications; American Mathematical Society: Providence, RI, USA, 2004; Volume 53.

24. Baier, S.; Zhao, L.Y. On Hecke eigenvalues at Piatetski-Shapiro primes. J. Lond. Math. Soc. 2010, 81, 175-201. [CrossRef]

25. Balog, A. On a variant of the Pjateckij-Šapiro prime number problem. In Groupe de Travail en Théorie Analytique et Élémentaire des Nombres, 1987-1988; Publ. Math. Orsay, 89-01; Université Paris-Saclay: Orsay, France, 1989; pp. 3-11.

26. Dufner, G. Pjateckij-Shapiro prime twins. J. Number Theory 1993, 43, 370-378. [CrossRef]

27. Zhang, D.Y.; Zhai, W.G. On the distribution of Hecke eigenvalues over Piatetski-Shapiro prime twins. Acta Math. Sin. (Engl. Ser.) 2021. [CrossRef]

28. Fomenko, O.M. Identities Involving Coefficients of Automorphic L-Functions. J. Math. Sci. 2006, 133, 1749-1755. [CrossRef]

29. Huang, J.; Liu, H.F.; Xu, F.X. Two-Dimensional Divisor Problems Related to Symmetric L-Functions, Symmetry 2021, 13, 359. [CrossRef]

30. Ichihara, Y. Estimates of a certain sum involving coefficients of cusp forms in weight and level aspects. Lith. Math. J. 2008, 48, 188-202. [CrossRef]

31. Jiang, Y.J.; Lü, G.S. Uniform estimates for sums of coefficients of symmetric square L-function. J. Number Theory 2015, 148, 220-234. [CrossRef]

32. Lao, H.X. On the fourth moment of coefficients of symmetric square L-function. Chin. Ann. Math. Ser. B 2012, 33, 877-888. [CrossRef]

33. Lü, G.S. Uniform estimates for sums of Fourier coefficients of cusp forms. Acta Math. Hungar. 2009, 124, 83-97. [CrossRef]

34. Sankaranarayanan, A. On a sum involving Fourier coefficients of cusp forms. Lithuanian Math. J. 2006, 46, 459-474. [CrossRef]

35. Tang, H.C. A note on the Fourier coefficients of Hecke Maass forms. J. Number Theory 2013, 133, 1156-1167. [CrossRef]

36. Zhang, R.; Han, X.; Zhang, D.Y. Power moments of the Riesz mean error term of symmetric square L-function in short intervals. Symmetry 2020, 12, 2036. [CrossRef]

37. Gallagher, P.X. A large sieve density estimate near $\sigma=1$. Invent. Math. 1970, 11, 329-339. [CrossRef]

38. Ye, Y.B.; Zhang, D.Y. Zero density for automorphic L-functions. J. Number Theory 2013, 133, 3877-3901. [CrossRef]

39. Dong, L.L.; Liu, H.F.; Zhang, D.Y. Zero density estimates for automorphic L-functions of SL(2, Z). Acta Math. Hungar. 2016, 148, 191-210. [CrossRef] 